\title{
Implementation of Gauss-Jackson Integration for Orbit Propagation'
}

\author{
Matthew M. Berry ${ }^{2}$ and Liam M. Healy ${ }^{3}$
}

\begin{abstract}
The Gauss-Jackson multi-step predictor-corrector method is widely used in numerical integration problems for astrodynamics and dynamical astronomy. The U.S. space surveillance centers have used an eighth-order Gauss-Jackson algorithm since the 1960s. In this paper, we explain the algorithm including a derivation from first principals and its relation to other multi-step integration methods. We also study its applicability to satellite orbits including its accuracy and stability.
\end{abstract}

\section{Introduction}

Determination and prediction of orbits requires an orbit propagator that finds the phase space state of a satellite at one time based on its state at another time. This function has traditionally been performed by an analytical computation such as Hamiltonian normalization (general perturbations). While numerical integration (special perturbations) can provide a wider range of force models, until recent years the computation time required made it prohibitive for routine use in space surveillance. With greatly increased computation speeds now widely available, the daily processing of 10,000 or more satellites routinely tracked by space surveillance centers can conceivably be based on numerical integration, and a significant fraction of this number are processed numerically now.

The Gauss-Jackson integrator [1] is a fixed-step predictor-corrector method. The Gauss-Jackson software used in space surveillance originated in the 1960s as part

${ }^{1}$ Based on paper AAS 01-426 presented at the AAS/AIAA Astrodynamics Specialists Conference, Quebec City, Canada, July 30-August 2, 2001.

${ }^{2}$ Astrodynamics Engineer, Analytical Graphics Inc., 220 Valley Creek Blvd., Exton, PA 19341. E-mail: mberry@agi.com. This work was completed while the first author was a Graduate Assistant in the Department of Aerospace and Ocean Engineering at Virginia Tech and an employee of the Naval Research Laboratory.

${ }^{3}$ Research Physicist, Naval Research Laboratory, Code 8233, Washington, DC 20375-5355. E-mail: Liam.Healy@nrl.navy.mil. 
of the Advanced Orbit/Estimation Subsystem (AOES) [2]. Over the years, AOES has given rise to a family of astrodynamics applications used in the various space surveillance centers whose integrators are essentially unchanged, including a system known as SpecialK [3]. SpecialK has been used by Naval Network and Space Operations Command for special perturbations catalog maintenance for the last six years. Another member of the family is Astrodynamics Support Workstation (ASW) [4], used in Cheyenne Mountain to support the special perturbations cata$\log$ and in support of conjunction assessment for NASA. We have chosen to use SpecialK as the implementation on which to focus, but our analysis has broad applicability to all Gauss-Jackson integrators, including the one in ASW. This paper presents a study of the theory upon which the integrator is based, how it is implemented, and its accuracy and stability.

After an overview of multi-step integrators, we start with an explanation of difference tables and operators that form the core of the derivation of all the multi-step methods. We apply these to differentiation in the next section, followed by single integration where we derive the nonsummed Adams methods. The Adams method has a summed form which is presented in the succeeding section. The next section, on the Störmer-Cowell integrator for second-order differential equations, makes use of the Adams results. The summed form of the Störmer-Cowell integrator, called the Gauss-Jackson integrator, is presented next. Having avoided the issue of how to start the integrator when backpoints are unavailable, we next address this issue, in which the concept of a mid-corrector is introduced. To this point, the formulation is in terms of the difference operators; for computational efficiency, the ordinate form sums like terms but makes the result order-specific. The details are included in the next section.

With the derivation of the integrators complete, we finish with two sections. The first is a detailed description of implementation complete with pseudo-code that should suffice for a reasonably skilled programmer to implement in any generalpurpose programming language. The second shows the effect of step size and order of the Gauss-Jackson integrator on accuracy and stability.

\section{Multi-Step Integrators}

An ordinary differential equation (ODE) is an equation of the form

$$
\frac{\mathrm{d} y}{\mathrm{~d} t}=f(t, y)
$$

in which the derivative of a dependent variable $y$ is a function $f$ of that variable and the independent variable $t$. In order to find a specific solution of a differential equation the initial conditions $\left(t_{0}, y\left(t_{0}\right)\right)$ must be given. A given differential equation along with initial conditions is known as an initial value problem. The solution to an initial value problem, $y(t)$, can be found analytically for some differential equations. However, most differential equations must be solved numerically. Algorithms that numerically solve initial value problems are known as numerical integrators. Numerical integrators give an approximate solution at distinct values of $t$, known as mesh points. The numerical solution at a mesh point $t_{n}$ is denoted $y_{n}$, and due to error in the numerical method will likely be different from the exact solution, $y\left(t_{n}\right)$.

The differential equation in (1) is called a first-order differential equation because the equation is for the first derivative. Differential equations may have a higher order 


$$
\frac{\mathrm{d}^{n} y}{\mathrm{~d} t^{n}}=f\left(t, y, y^{\prime}, \ldots, y\right)^{(n-1)}
$$

where $n$ is the order of the differential equation. Initial value problems involving higher-order ODEs require initial conditions for each derivative through $y^{(n-1)}$. Though higher-order ODEs may be rewritten as a system of first order equations and solved with a standard numerical integrator, some numerical integrators are designed to directly solve higher-order ODEs. Integrators that solve second-order ODEs are called double-integration methods, while integrators that solve first-order ODEs are called single-integration methods.

Multi-step integrators integrate forward from a particular time to the next mesh point using function values at the current point as well as several previous mesh points. The set of the previous points used as well as the current point is called the set of backpoints. One disadvantage of multi-step integrators is that the initial set of backpoints must be found through some startup procedure, which complicates the method. The methods develop a Taylor series in the time separation between mesh points, and this series must be truncated after some number of terms, which thereby gives the order of the method. The order is one less than the number of backpoints, and is not related to the order of the differential equation.

Multi-step integrators are known as predictor-corrector methods. The algorithms first predict a $y$ value for the next mesh point and the function $f(t, y)$ is evaluated at the predicted point. That predicted function value is added to the set of backpoints, and a corrector formula is used with this revised set of backpoints to refine the predicted $y$ value.

This procedure offers several variations on the implementation. First, when adding the predicted value to the set of backpoints, the farthest backpoint from the current point may or may not be dropped. If it is not dropped, the corrector is of higher order than the predictor because the corrector uses one more backpoint than the predictor. Otherwise, the predictor and corrector are of the same order. Also, a second evaluation may or may not be performed after the corrector. A second evaluation improves the accuracy of the method, because improved function values are used in the set of backpoints in the subsequent steps. However, the additional evaluation causes an increase in run time. Methods that use one evaluation per step are known as Predict-Evaluate-Correct, or PEC, methods, and methods that perform a second evaluation are called PECE methods. Some implementations perform additional iterations of the corrector to meet some tolerance, and are called $\mathrm{P}(\mathrm{EC})^{n}$ or $\mathrm{PE}(\mathrm{CE})^{n}$ methods.

The methods can be derived in a variety of ways, but the fixed-step methods can be derived in a simplified form by using backward difference operators, which are described in the next section. The derivations of the Adams, summed-Adams, Störmer-Cowell, and Gauss-Jackson methods follow. These derivations follow the derivations presented by Maury and Segal [5], and the NORAD document known as TP008 [6].

The function $f(t, y)$ must be continuous and smooth through the set of backpoints. If there are any discontinuities in $f$, for example going through eclipse when solar radiation pressure is considered, the integration must either be restarted, or modified to handle the discontinuity [7].

In this paper we do not address variable step or error control, including $s$-integration, in numerical integration. The interested reader is referred to [8] and [9] for information on this topic. 


\section{Tables and Operators}

Predictor-corrector integrators can be defined in terms of difference tables. For a fixed-step method, assume that solution values $y_{n}$ are known on a discrete set of equally-spaced mesh points, $\ldots, t_{0}-2 h, t_{0}-h, t_{0}, t_{0}+h, t_{0}+2 h, \ldots$, where $h$ is the step. As shorthand, these values are referred to as $t_{n}=t_{0}+n h$, so this set of five values is $t_{-2}, t_{-1}, t_{0}, t_{1}, t_{2}$. The corresponding function values are $f_{n}=f\left(t_{n}, y_{n}\right)$, where $y_{n}$ is the numerical solution at the mesh point $t_{n}$ (not the exact solution $y\left(t_{n}\right)$ ).

The differential equations are solved by taking differences of the function values. There are three kinds of differences, represented by different operators:

$$
\begin{array}{ll}
\text { forward difference } & \Delta f_{i}=f_{i+1}-f_{i} \\
\text { backward difference } & \nabla f_{i}=f_{i}-f_{i-1} \\
\text { central difference } & \delta f_{i}=f_{i+1 / 2}-f_{i-1 / 2}
\end{array}
$$

Note that first central differences cannot be calculated directly, as the half-step points are not in the sequence of points, though the second central differences (see below for definition of second differences) can be calculated. As previous authors ([10], [11]) have recognized, conversion between series in these operators is straightforward. The backward difference derivation is used here, because when one is predicting or correcting, i.e., any time other than startup, it is the most natural. Derivations using other difference operators give algebraically equivalent methods, though differences in their implementation may give slightly different results due to round-off error.

Not only can the differences of the function $f$ be computed, the differences of the differences, or second differences, can be computed, and so on. For instance, the square of the backward difference operator is the operator applied to the first difference

$$
\begin{aligned}
\nabla^{2} f_{i} & =\nabla \nabla f_{i}=\nabla\left(f_{i}-f_{i-1}\right) \\
& =f_{i}-f_{i-1}-\left(f_{i-1}-f_{i-2}\right)=f_{i}-2 f_{i-1}+f_{i-2}
\end{aligned}
$$

The relationships of the backward differences are illustrated in Table 1. The arrows in the table point towards the difference; the upper component is always subtracted from the lower. For example, $\nabla^{2} f_{1}=\nabla f_{1}-\nabla f_{0}$.

In addition to the three difference operators, there is also a displacement operator $E$. The displacement operator is defined as

$$
E f_{i}=f_{i+1}
$$

Powers of this operator act as expected; e.g., $E^{2} f_{i}=f_{i+2}$. The backward difference operator can be defined in terms of the displacement operator

$$
\nabla=1-E^{-1}
$$

and alternatively the displacement operator can be defined in terms of the backward difference operator

$$
E=\frac{1}{1-\nabla}
$$

The summed integration formulas (see Summed Adams Method section), contain negative powers of the operators; e.g., $\nabla^{-2}$. The backward difference table 
TABLE 1. Backward Difference Table

\begin{tabular}{|c|c|c|c|c|}
\hline$i$ & $f_{i}$ & $\nabla f_{i}$ & $\nabla^{2} f_{i}$ & $\nabla^{3} f_{i}$ \\
\hline-3 & $f_{-3}$ & $\nabla f_{-3}$ & $\nabla^{2} f_{-3}$ & $\nabla^{3} f_{-3}$ \\
\hline-2 & $f_{-2}$ & $\nabla f_{-2}$ & & $\nabla^{3} f_{-2}$ \\
\hline-1 & $f_{-1}$ & $\nabla f_{-1}$ & $\nabla^{2} f_{-1}$ & $\nabla^{3} f_{-1}$ \\
\hline 0 & $f_{0}$ & $\nabla f_{0}$ & $\nabla^{2} f_{0}$ & $\nabla^{3} f_{0}$ \\
\hline 1 & $f_{1}$ & $\nabla f_{1}$ & $\nabla^{2} f_{1}$ & $\nabla^{3} f_{1}$ \\
\hline 2 & $f_{2}$ & $\nabla f_{2}$ & $\nabla^{2} f_{2}$ & $\nabla^{3} f_{2}$ \\
\hline 3 & $f_{3}$ & $\nabla f_{3}$ & $\nabla^{2} f_{3}$ & $\nabla^{3} f_{3}$ \\
\hline
\end{tabular}

(Table 1) can be extended to the left to get negative powers of the backward difference operator, as in Table 2. Extending the differencing to the left gives $f_{i}=\nabla^{-1} f_{i}-\nabla^{-1} f_{i-1}$. This relation can be changed into a recursion formula that defines the inverse operator

$$
\nabla^{-1} f_{i}=f_{i}+\nabla^{-1} f_{i-1}
$$

Note that the initial $(i=0)$ term in the sum is arbitrary; the difference relation holds no matter what this value is. This value is effectively an integration constant $C_{1}$. Thus, if the inverse operator is defined as a sum

$$
\nabla^{-1} f_{1}= \begin{cases}C_{1}+\sum_{j=1}^{i} f_{j}, & \text { if } i \geq 1 \\ C_{1}-\sum_{j=i+1}^{0} f_{j}, & \text { if } i \leq-1\end{cases}
$$

TABLE 2. Inverse Backward Differences

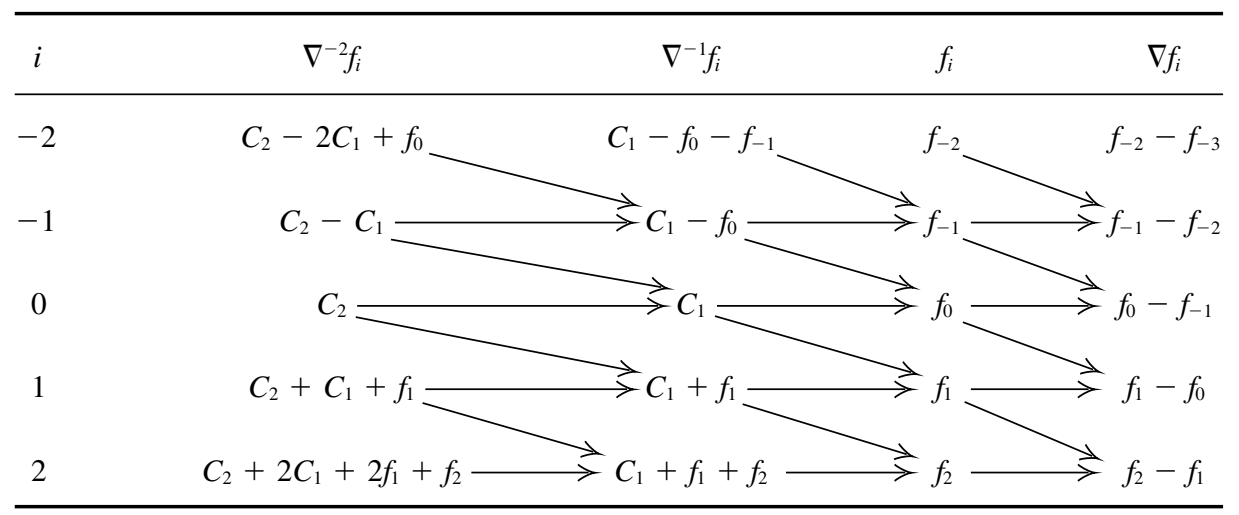


the difference relation holds. The constant $C_{1}$ is discussed further in the Implementation section.

Powers of the summation $\nabla^{-1}$ are understood to be multiple sums, analogous to multiple differences. The presence of the operator $\nabla^{-2}$ in the Gauss-Jackson formulas sometimes gives rise to the name second sum integration formula. The second sum has an integration constant $C_{2}$, analogous to $C_{1}$.

\section{Differentiation}

Differentiation $D=\mathrm{d} / \mathrm{d} t$ is the operator that turns a function into its derivative function. The differentiation operator $D$ can be represented in terms of $E$ by noting that

$$
\begin{aligned}
E^{p} f\left(t_{0}\right) & =f\left(t_{0}+p h\right) \\
& =f\left(t_{0}\right)+p h D f\left(t_{0}\right)+\frac{(p h)^{2}}{2 !} D^{2} f\left(t_{0}\right)+\frac{(p h)^{3}}{3 !} D^{3} f\left(t_{0}\right)+\ldots \\
& =e^{p h D} f\left(t_{0}\right)
\end{aligned}
$$

for any real number $p$, using a Taylor series expansion. The exponential of an operator is to be interpreted as its ordinary Taylor expansion

$$
e^{t}=1+t+\frac{t^{2}}{2 !}+\frac{t^{3}}{3 !}+\ldots
$$

with powers of the operator well-defined. Thus we may identify the two operators

$$
E^{p}=e^{p h D}
$$

or taking the logarithm

$$
p h D=p \log E
$$

This expression can be written in terms of the backward difference operator (6)

$$
h D=\log E=-\log (1-\nabla)
$$

The integration methods can now be derived by taking the inverse of the differentiation operator. The Adams method is derived first, and the other methods are derived from it.

\section{Adams Method}

Indefinite integration is the operator inverse of differentiation

$$
\int \mathrm{d} t=D^{-1}
$$

so its action on an arbitrary function is to give the antiderivative

$$
F(t)=D^{-1} f(t)
$$

The single-integration operator $D^{-1}$ is computed as the inverse of the differentiation operator (13)

$$
D^{-1}=-\frac{h}{\log (1-\nabla)}
$$

With this operator Adams integration can be developed. The focus here is satellite orbits, so the function $f$ is replaced with acceleration $\ddot{\mathbf{r}}$. 
The definite integral is computed by using the displacement operator on the indefinite integral

$$
\left(E^{p}-1\right) D^{-1} \ddot{\mathbf{r}}_{\mathrm{n}}=\left(E^{p}-1\right) \dot{\mathbf{r}}_{\mathrm{n}}=\int_{t_{n}}^{t_{n}+p h} \ddot{\mathbf{r}} \mathrm{d} t
$$

For one step, $p$ is 1 . This integration corresponds to a predictor in a multi-step numerical integration, because the equation finds a value of $\dot{\mathbf{r}}$ at a future time. If $p=1$, the predictor operator $J$ can be written in terms of the backward difference operator as

$$
\begin{aligned}
J & =\frac{1}{h}(E-1) D^{-1}=\frac{1}{h}\left[(1-\nabla)^{-1}-1\right] D^{-1}=\frac{1}{h} \frac{\nabla}{(1-\nabla)} D^{-1} \\
& =-\frac{\nabla}{(1-\nabla) \log (1-\nabla)}
\end{aligned}
$$

To integrate from $t_{n}-h$ to $t_{n}$, shift backward using the displacement operator (6)

$$
E^{-1} J=(1-\nabla) J=-\frac{\nabla}{\log (1-\nabla)}
$$

This operation corresponds to the corrector in a multi-step numerical integration, because it assumes that the $n^{\text {th }}$ velocity is already known and uses the corresponding acceleration to recalculate a new, better, velocity. It is on this expression that we focus initially because the operator has the simplest expansion.

The coefficients of the operators may be developed using a recursion relation [12]. For convenience in developing expansions, we define an operator $L$ and its Taylor expansion with coefficients $c_{i}$ as

$$
L=E^{-1} J=-\frac{\nabla}{\log (1-\nabla)}=c_{0}+c_{1} \nabla+c_{2} \nabla^{2}+c_{3} \nabla^{3}+\ldots
$$

We shall develop the expansion of $L$ recursively through the standard Taylor series of the logarithm

$$
\log (1-\nabla)=-\nabla-\frac{1}{2} \nabla^{2}-\frac{1}{3} \nabla^{3}-\frac{1}{4} \nabla^{4} \ldots
$$

which can be substituted in the definition of $L$

$$
L=-\frac{\nabla}{\log (1-\nabla)}=\frac{1}{1+\frac{1}{2} \nabla+\frac{1}{3} \nabla^{2}+\frac{1}{4} \nabla^{3}+\ldots}=\sum_{i=0}^{\infty} c_{i} \nabla^{i}
$$

and then expanded in the unknowns $c_{i}$

$$
\left(c_{0}+c_{1} \nabla+c_{2} \nabla^{2}+c_{3} \nabla^{3}+\ldots\right)\left(1+\frac{1}{2} \nabla+\frac{1}{3} \nabla^{2}+\frac{1}{4} \nabla^{3}+\ldots\right)=1
$$

Expanding and grouping by powers of $\nabla$

$$
\begin{gathered}
c_{0}+\left(\frac{1}{2} c_{0}+c_{1}\right) \nabla+\left(\frac{1}{3} c_{0}+\frac{1}{2} c_{1}+c_{2}\right) \nabla^{2} \\
+\left(\frac{1}{4} c_{0}+\frac{1}{3} c_{1}+\frac{1}{2} c_{2}+c_{3}\right) \nabla^{3}+\ldots=1
\end{gathered}
$$


allows for the development of a recursion relation for the coefficients

$$
\begin{aligned}
& 1=c_{0} \\
& 0=\frac{1}{2} c_{0}+c_{1} \\
& 0=\frac{1}{3} c_{0}+\frac{1}{2} c_{1}+c_{2}
\end{aligned}
$$

or, in general

$$
c_{n}=-\sum_{i=0}^{n-1} \frac{c_{i}}{n+1-i}
$$

for $n \geq 1$ with $c_{0}=1$. The first few coefficients are

\begin{tabular}{c|ccccccccc}
$i$ & 0 & 1 & 2 & 3 & 4 & 5 & 6 & 7 & 8 \\
\hline$c_{i}$ & 1 & $-\frac{1}{2}$ & $-\frac{1}{12}$ & $-\frac{1}{24}$ & $-\frac{19}{720}$ & $-\frac{3}{160}$ & $-\frac{863}{60480}$ & $-\frac{275}{24192}$ & $-\frac{33953}{3628800}$
\end{tabular}

With the coefficients $c_{i}$ known, integration from $t_{n}-h$ to $t_{n}$ may be written in terms of $L$ as

$$
\int_{t_{n}-h}^{t_{n}} \ddot{\mathbf{r}} \mathrm{d} t=h E^{-1} J \ddot{\mathbf{r}}_{\mathrm{n}}=h L \ddot{\mathbf{r}}_{\mathrm{n}}
$$

A formula for the corrected value can be found by performing the integration and using the coefficients $c_{i}$

$$
\dot{\mathbf{r}}_{\mathrm{n}}=\dot{\mathbf{r}}_{\mathrm{n}-1}+h\left(1-\frac{1}{2} \nabla-\frac{1}{12} \nabla^{2}-\frac{1}{24} \nabla^{3}-\frac{19}{720} \nabla^{4}-\frac{3}{160} \nabla^{5}+\ldots\right) \dot{\mathbf{r}}_{\mathrm{n}}
$$

This formula is the Adams-Moulton corrector formula in difference form. This form is called the difference form because it uses the differences $\nabla^{i}$. An alternate form, called the ordinate form, in which the differences are rewritten in terms of the function values, is described in the Ordinate Forms section.

Returning to the predictor (18), the expansion of $J$ can be computed by noting that

$$
J=(1-\nabla)^{-1} L=\left(1+\nabla+\nabla^{2}+\ldots\right)\left(c_{0}+c_{1} \nabla+c_{2} \nabla^{2}+\ldots\right)=\sum_{i=0}^{\infty} \gamma_{i} \nabla^{i}
$$

Each coefficient $\gamma_{i}$ can be expressed as the sum of the coefficients $c_{k}, k \leq i$

$$
\gamma_{i}=\sum_{k=0}^{i} c_{k}
$$

so they may be computed directly from (27)

\begin{tabular}{c|ccccccccc}
$i$ & 0 & 1 & 2 & 3 & 4 & 5 & 6 & 7 & 8 \\
\hline$\gamma_{i}$ & 1 & $\frac{1}{2}$ & $\frac{5}{12}$ & $\frac{3}{8}$ & $\frac{251}{720}$ & $\frac{95}{288}$ & $\frac{19087}{60480}$ & $\frac{5257}{17280}$ & $\frac{1070017}{3628800}$
\end{tabular}


and the integral is then

$$
\int_{t_{n}}^{t_{n}+h} \ddot{\mathbf{r}} \mathrm{d} t=h J \ddot{\mathbf{r}}_{\mathrm{n}}
$$

or

$$
\dot{\mathbf{r}}_{\mathrm{n}+1}=\dot{\mathbf{r}}_{\mathrm{n}}+h\left(1+\frac{1}{2} \nabla+\frac{5}{12} \nabla^{2}+\frac{3}{8} \nabla^{3}+\frac{251}{720} \nabla^{4}+\frac{95}{288} \nabla^{5}+\ldots\right) \ddot{\mathbf{r}}_{\mathrm{n}}
$$

This formula is the Adams-Bashforth predictor formula in difference form, so called because when applied to the velocity and acceleration at $t_{n}$ it finds the velocity at the next mesh point $t_{n+1}$.

In practice, given $m$ known accelerations

$$
\ddot{\mathbf{r}}_{\mathrm{n}-\mathrm{m}+1}, \ldots, \ddot{\mathbf{r}}_{\mathrm{n}}
$$

the backward difference table (Table 1) for the derivatives can be computed through $\nabla^{m-1}$, a predicted value for $\dot{\mathbf{r}}_{\mathrm{n}+1}$ is made based on (34), then that value is corrected with (29).

Although our purpose in deriving the nonsummed Adams-Bashforth and AdamsMoulton coefficients is to use these results in the Gauss-Jackson method, these formulae stand on their own as a widely-used numerical integration method of first-order differential equations. For greater accuracy, summed methods may be preferable, and we treat the summed form of the Adams method next.

\section{Summed Adams Method}

An alternative form of single integration, called the summed form, can be developed using the summation operator $\nabla^{-1}$. The Adams-Moulton corrector formula (29) may be written so both velocity terms are on the left side

$$
\dot{\mathbf{r}}_{\mathrm{n}}-\dot{\mathbf{r}}_{\mathrm{n}-1}=h\left(1-\frac{1}{2} \nabla-\frac{1}{12} \nabla^{2}-\frac{1}{24} \nabla^{3}-\frac{19}{720} \nabla^{4}-\frac{3}{160} \nabla^{5}+\ldots\right) \ddot{\mathbf{r}}_{\mathrm{n}}
$$

The difference of the two velocity terms may be written using the backward difference operator

$$
\nabla \dot{\mathbf{r}}_{\mathrm{n}}=h\left(1-\frac{1}{2} \nabla-\frac{1}{12} \nabla^{2}-\frac{1}{24} \nabla^{3}-\frac{19}{720} \nabla^{4}-\frac{3}{160} \nabla^{5}+\ldots\right) \ddot{\mathbf{r}}_{\mathrm{n}}
$$

The corrected value of the velocity can be found by applying $\nabla^{-1}$ to both sides of (37), [5]

$$
\dot{\mathbf{r}}_{\mathrm{n}}=h\left(\nabla^{-1}-\frac{1}{2}-\frac{1}{12} \nabla-\frac{1}{24} \nabla^{2}-\frac{19}{720} \nabla^{3}-\frac{3}{160} \nabla^{4}+\ldots\right) \ddot{\mathbf{r}}_{\mathrm{n}}
$$

This expression is the summed Adams corrector formula. The summed form uses the same coefficients as the Adams-Moulton corrector, but they have been shifted by one place. The formula is called the summed form because of the presence of the summation operator. 
A similar process can be performed on the Adams-Bashforth predictor formula (34)

$$
\dot{\mathbf{r}}_{\mathrm{n}+1}=h\left(\nabla^{-1}+\frac{1}{2}+\frac{5}{12} \nabla+\frac{3}{8} \nabla^{2}+\frac{251}{720} \nabla^{3}+\frac{95}{288} \nabla^{4}+\ldots\right) \ddot{\mathbf{r}}_{\mathrm{n}}
$$

giving the summed Adams predictor. The summed form gives the predicted or corrected value by integrating directly from epoch, while the nonsummed form integrates from point to point. According to Henrici ([13], p. 327) the summed form is preferred for reducing the propagation of roundoff error.

\section{Störmer-Cowell}

To find a corrector formula for position, the corrector may be applied to the Adams-Moulton corrector (28)

$$
h L \int_{t_{n}-h}^{t_{n}} \ddot{\mathbf{r}} \mathrm{d} t=h L\left(\dot{\mathbf{r}}_{\mathrm{n}}-\dot{\mathbf{r}}_{\mathrm{n}-1}\right)=h^{2} L^{2} \ddot{\mathbf{r}}_{\mathrm{n}}
$$

Application of the corrector to both velocity terms, e.g., $h L \dot{\mathbf{r}}_{\mathrm{n}}=\mathbf{r}_{\mathrm{n}}-\mathbf{r}_{\mathrm{n}-1}$, gives an expression for position

$$
\mathbf{r}_{\mathrm{n}}=2 \mathbf{r}_{\mathrm{n}-1}-\mathbf{r}_{\mathrm{n}-2}+h^{2} L^{2} \ddot{\mathbf{r}}_{\mathrm{n}}
$$

To find the coefficients in the expansion of $L^{2}$

$$
L^{2}=q_{0}+q_{1} \nabla+q_{2} \nabla^{2}+\ldots
$$

in terms of the Adams coefficients $c$ (26), note that (42) is the square of (20)

$$
q_{i}=\sum_{k=0}^{i} c_{k} c_{i-k}
$$

so the first nine coefficients are

\begin{tabular}{c|ccccccccc}
$i$ & 0 & 1 & 2 & 3 & 4 & 5 & 6 & 7 & 8 \\
\hline$q_{i}$ & 1 & -1 & $\frac{1}{12}$ & 0 & $-\frac{1}{240}$ & $-\frac{1}{240}$ & $-\frac{221}{60480}$ & $-\frac{19}{6048}$ & $-\frac{9829}{3628800}$
\end{tabular}

These are the Cowell corrector coefficients. The corrector formula is given by using these coefficients in (41)

$$
\mathbf{r}_{\mathrm{n}}=2 \mathbf{r}_{\mathrm{n}-1}-\mathbf{r}_{\mathrm{n}-2}+h^{2}\left(1-\nabla+\frac{1}{12} \nabla^{2}-\frac{1}{240} \nabla^{4}-\frac{1}{240} \nabla^{5}+\ldots\right) \ddot{\mathbf{r}}_{\mathrm{n}}
$$

This formula is the Cowell corrector formula [5]. It is a double-integration formula, since it computes the position given acceleration.

As with single integration, the predictor formula is found by shifting the step at which the operator is applied, in other words applying the displacement operator $E$ to both sides of (41)

$$
\mathbf{r}_{\mathrm{n}+1}=2 \mathbf{r}_{\mathrm{n}}-\mathbf{r}_{\mathrm{n}-1}+h^{2} E L^{2} \ddot{\mathbf{r}}_{\mathrm{n}}
$$

Writing the displacement operator $E$ in terms of $\nabla,(6)$, and applying to $L^{2}$

$$
E L^{2}=(1-\nabla)^{-1} L^{2}=\left(1+\nabla+\nabla^{2}+\ldots\right)\left(q_{0}+q_{1} \nabla+q_{2} \nabla^{2}+\ldots\right)=\sum_{i=1}^{\infty} \lambda_{i} \nabla^{i}
$$


shows that the predictor coefficients $\lambda_{i}$ can be written as a sum of the corrector coefficients, just as with single integration. The predictor coefficients can be computed from (44)

\begin{tabular}{c|ccccccccc}
$i$ & 0 & 1 & 2 & 3 & 4 & 5 & 6 & 7 & 8 \\
\hline$\lambda_{i}$ & 1 & 0 & $\frac{1}{12}$ & $\frac{1}{12}$ & $\frac{19}{240}$ & $\frac{3}{40}$ & $\frac{863}{12096}$ & $\frac{275}{4032}$ & $\frac{33953}{518400}$
\end{tabular}

Using these coefficients in (46)

$$
\mathbf{r}_{\mathrm{n}+1}=2 \mathbf{r}_{\mathrm{n}}-\mathbf{r}_{\mathrm{n}-1}+h^{2}\left(1+\frac{1}{12} \nabla^{2}+\frac{1}{12} \nabla^{3}+\frac{19}{240} \nabla^{4}+\frac{3}{40} \nabla^{5}+\ldots\right) \ddot{\mathbf{r}}_{\mathrm{n}}
$$

gives the Störmer predictor formula [5].

\section{Gauss-Jackson}

The Gauss-Jackson method is the summed form of the Störmer-Cowell method. According to Herrick ([10], p. 12), the method was named Gauss-Jackson because of its appearance in a 1924 paper by Jackson [1]. The Gauss-Jackson method is also referred to as the second-sum method. Sometimes, the name is associated with a central difference derivation, because that is how Jackson showed it. However, as we mentioned, the derivation by any of the difference operators are equivalent; what is unique about Gauss-Jackson is the presence of the second sum.

The Gauss-Jackson corrector can be derived from the Cowell corrector by noting that the position terms in (45) may be combined using a second backward difference

$$
\nabla^{2} \mathbf{r}_{\mathrm{n}}=h^{2}\left(1-\nabla+\frac{1}{12} \nabla^{2}-\frac{1}{240} \nabla^{4}-\frac{1}{240} \nabla^{5}-\frac{221}{60480} \nabla^{6}+\ldots\right) \ddot{\mathbf{r}}_{\mathrm{n}}
$$

Applying the second sum $\nabla^{-2}$ to both sides gives an equation for position [5]

$$
\mathbf{r}_{\mathrm{n}}=h^{2} \nabla^{-2}\left(1-\nabla+\frac{1}{12} \nabla^{2}-\frac{1}{240} \nabla^{4}-\frac{1}{240} \nabla^{5}-\frac{221}{60480} \nabla^{6}+\ldots\right) \ddot{\mathbf{r}}_{\mathrm{n}}
$$

Note that $(1-\nabla) \ddot{\mathbf{r}}_{\mathrm{n}}=E^{-1} \ddot{\mathbf{r}}_{\mathrm{n}}=\ddot{\mathbf{r}}_{\mathrm{n}-1}$, so the first two terms can be simplified to

$$
\mathbf{r}_{\mathrm{n}}=h^{2}\left[\nabla^{-2} \ddot{\mathbf{r}}_{\mathrm{n}-1}+\left(\frac{1}{12}-\frac{1}{240} \nabla^{2}-\frac{1}{240} \nabla^{3}-\frac{221}{60480} \nabla^{4}+\ldots\right) \ddot{\mathbf{r}}_{\mathrm{n}}\right]
$$

This expression is the Gauss-Jackson corrector formula.

A similar process on the Störmer predictor (49) gives a summed predictor

$$
\mathbf{r}_{\mathrm{n}+1}=h^{2}\left[\nabla^{-2} \ddot{\mathbf{r}}_{\mathrm{n}}+\left(\frac{1}{12}+\frac{1}{12} \nabla+\frac{19}{240} \nabla^{2}+\frac{3}{40} \nabla^{3}+\ldots\right) \ddot{\mathbf{r}}_{\mathrm{n}}\right]
$$

which is the Gauss-Jackson predictor formula. Note that for the predictor the second sum operators acts on $\ddot{\mathbf{r}}_{\mathrm{n}}$, while for the corrector it acts on $\ddot{\mathbf{r}}_{\mathrm{n}-1}$.

\section{Startup Formulas}

In order to calculate the differences $\nabla^{i}$, the difference table (Table 1 ) must be calculated, which depends on having values of acceleration at the backpoints. To calculate the $N^{\text {th }}$ difference $N+1$ points must be known. The initial value problem 
only gives the initial conditions at epoch, so a startup procedure is required to find position and velocity, and then acceleration, at the other backpoints. One possible method is to use a single-step integrator, such as Runge-Kutta, to find the values at the initial set of backpoints. Alternatively, one may use the two-body motion solution, which may be computed analytically. In the latter case particularly, we use an iterative method that takes an initial guess of the backpoints and refines them with corrector formulas. These corrector formulas, which correct a value using not only previous points, but also future known points, may be called mid-corrector formulas.

Where two-body motion is close to the actual motion modeled, the initial set of guess values can be found by using the analytic two-body solution. In other cases, a good analytic approximation of the full motion would be necessary. For a method that uses the $N^{\text {th }}$ difference, $N+1$ total points are needed, so $N$ points in addition to epoch must be found. To reduce the error that comes from the two-body solution, instead of propagating $N$ steps forward, $N / 2$ steps are taken both forward and backward. These points are numbered using a symmetric index, from $-N / 2$ to $N / 2$, with epoch numbered 0 . This system requires that $N$ be even, as it is for the integrators in this study. If $N$ were odd an additional point would be needed either before or after epoch.

After the set of guess values is found, each value other than epoch is corrected with a mid-corrector formula, except for the last value, which uses the corrector formula. The formulas are numbered using the letter $j$ as an index with symmetric index numbers. So the mid-correctors are numbered $j=-N / 2$ to $j=N / 2-1$, and the corrector is numbered $j=N / 2$. Similarly the predictor can be considered the $j=N / 2+1$ formula.

The mid-correctors are found from the corrector by applying the inverse displacement operator $E^{-1}=1-\nabla$ repeatedly. Using the corrector operator $L$ as an example, the relation of operators to coefficient sets can be seen on the diagram for an eighth-order method $(N=8)$ :

$$
\text { mid-correctors } \quad\left\{\begin{array}{ccc}
j=-4 & (1-\nabla)^{8} L & \\
j=-3 & (1-\nabla)^{7} L & \\
\vdots & \vdots & \uparrow \text { apply } 1-\nabla \\
j=2 & (1-\nabla)^{2} L & \downarrow \text { apply }(1-\nabla)^{-1} \\
j=3 & (1-\nabla) L & \\
\text { corrector } & j=4 & L \\
\text { predictor } & j=5 & \frac{L}{1-\nabla}
\end{array}\right.
$$

The coefficients for each mid-corrector may be computed from the next one by application of the operator $1-\nabla$. For an arbitrary power series in $\nabla$ with coefficients $d$, multiply the series

$$
\left(d_{0}+d_{1} \nabla+d_{2} \nabla^{2}+\ldots\right)(1-\nabla)=d_{0}+\left(d_{1}-d_{0}\right) \nabla+\left(d_{2}-d_{1}\right) \nabla^{2}+\ldots
$$

In other words, the coefficients for a particular value of $j$ are just the differences of coefficients of adjacent values of powers of $\nabla$ for the next higher value of $j$. As shown in the derivation of the Adams and Störmer-Cowell predictors from their 
correctors, the coefficients are also the sums of all the coefficients of the next lower $j$, through the particular power of $\nabla$

$$
\begin{aligned}
\left(m_{0}\right. & \left.+m_{1} \nabla+m_{2} \nabla^{2}+\ldots\right) \frac{1}{1-\nabla} \\
& =\left(m_{0}+m_{1} \nabla+m_{2} \nabla^{2}+\ldots\right)\left(1+\nabla+\nabla^{2}+\nabla^{3}+\ldots\right) \\
& =\sum_{k}\left(\sum_{i=0}^{k} m_{i}\right) \nabla^{k}
\end{aligned}
$$

All the coefficients, mid-correctors, corrector, and predictor, taken together may be viewed as an array, with the rows indexed by $j$, and the columns by the exponent $i$ of $\nabla$. For an $N^{\mathrm{th}}$-order integrator, there are $N+1$ terms in the series expansion of the operator for each $j$, and there are $N+2$ values of $j$; therefore, the full array of coefficients has $(N+1)(N+2)$ elements. For example, the eighth-order integrator has $9 \times 10=90$ coefficients.

The summed Adams corrector formula (38) can be written using the coefficient array $\beta$

$$
\dot{\mathbf{r}}_{\mathrm{n}}=h\left(\nabla^{-1} \ddot{\mathbf{r}}_{\mathrm{n}}+\sum_{i=0}^{N} \beta_{\frac{N}{2}, i} \nabla^{i} \ddot{\mathbf{r}}_{\mathrm{n}}\right)
$$

From this, the last mid-corrector formula $\left(j=\frac{N}{2}-1\right)$ is obtained from the coefficient differences (54) of the corrector

$$
\begin{aligned}
\dot{\mathbf{r}}_{\frac{N}{2}-1} & =E^{-1} \dot{\mathbf{r}}_{\frac{N}{2}} \\
& =E^{-1} h\left[\nabla^{-1} \ddot{\mathbf{r}}_{\frac{N}{2}}+\left(-\frac{1}{2}-\frac{1}{12} \nabla-\frac{1}{24} \nabla^{2}-\frac{19}{720} \nabla^{3}-\frac{3}{160} \nabla^{4}+\ldots\right) \dot{\mathbf{r}}_{\frac{N}{2}}\right] \\
& =h\left[\nabla^{-1} \dot{\mathbf{r}}_{\frac{N}{2}-1}+\left(-\frac{1}{2}+\frac{5}{12} \nabla+\frac{1}{24} \nabla^{2}+\frac{11}{720} \nabla^{3}+\frac{11}{1440} \nabla^{4}+\ldots\right) \ddot{\mathbf{r}}_{\frac{N}{2}}\right]
\end{aligned}
$$

The general mid-corrector formulas can be written as

$$
\dot{\mathbf{r}}_{\mathrm{n}}=h\left(\nabla^{-1} \ddot{\mathbf{r}}_{\mathrm{n}}+\sum_{i=0}^{N} \beta_{n i} \nabla^{i} \dot{\mathbf{r}}_{\frac{\mathrm{N}}{2}}\right)
$$

where $-\frac{N}{2} \leq n \leq \frac{N}{2}-1$. The coefficients $\beta_{j i}$ for each value of $j$ are obtained by taking differences of the coefficients for $j+1$. The mid-corrector coefficients are thus computed recursively

$$
\beta_{j i}= \begin{cases}\beta_{j+1, i}-\beta_{j+1, i-1} & \text { if } i>0 \\ \beta_{j+1, i} & \text { if } i=0\end{cases}
$$

for $-\frac{N}{2} \leq j \leq \frac{N}{2}-1$

The predictor formula (39) may be rewritten in terms of the coefficient array $\beta$

$$
\dot{\mathbf{r}}_{\mathrm{n}+1}=h\left(\nabla^{-1} \ddot{\mathbf{r}}_{\mathrm{n}}+\sum_{i=0}^{N} \beta_{\frac{N}{2}+1, i} \nabla^{i} \ddot{\mathbf{r}}_{\mathrm{n}}\right)
$$

The eighth-order coefficients $\beta_{j i}$ are presented in Table 3 . 
TABLE 3. Eighth-Order Summed-Adams Difference Coefficients, $\beta_{j i}$

\begin{tabular}{cccccccccc}
\hline & \multicolumn{8}{c}{$i$} \\
\cline { 2 - 9 }$j$ & 0 & 1 & 2 & 3 & 4 & 5 & 6 & 7 & 8 \\
\hline-4 & $-\frac{1}{2}$ & $\frac{47}{12}$ & $-\frac{107}{8}$ & $\frac{18701}{720}$ & $-\frac{45083}{1440}$ & $\frac{1445281}{60480}$ & $-\frac{1354079}{120960}$ & $\frac{10468447}{3628800}$ & $-\frac{25713}{89600}$ \\
-3 & $-\frac{1}{2}$ & $\frac{41}{12}$ & $-\frac{239}{24}$ & $\frac{11531}{720}$ & $-\frac{22021}{1440}$ & $\frac{520399}{60480}$ & $-\frac{11603}{4480}$ & $\frac{1070017}{3628800}$ & $\frac{8183}{1036800}$ \\
-2 & $-\frac{1}{2}$ & $\frac{35}{12}$ & $-\frac{169}{24}$ & $\frac{6461}{720}$ & $-\frac{1011}{160}$ & $\frac{138241}{60480}$ & $-\frac{5257}{17280}$ & $-\frac{33953}{3628800}$ & $-\frac{425}{290304}$ \\
-1 & $-\frac{1}{2}$ & $\frac{29}{12}$ & $-\frac{37}{8}$ & $\frac{3131}{720}$ & $-\frac{2837}{1440}$ & $\frac{19087}{60480}$ & $\frac{275}{24192}$ & $\frac{7297}{3628800}$ & $\frac{7}{12800}$ \\
0 & $-\frac{1}{2}$ & $\frac{23}{12}$ & $-\frac{65}{24}$ & $\frac{1181}{720}$ & $-\frac{95}{288}$ & $-\frac{863}{60480}$ & $-\frac{13}{4480}$ & $-\frac{3233}{3628800}$ & $-\frac{2497}{7257600}$ \\
1 & $-\frac{1}{2}$ & $\frac{17}{12}$ & $-\frac{31}{24}$ & $\frac{251}{720}$ & $\frac{3}{160}$ & $\frac{271}{60480}$ & $\frac{191}{120960}$ & $\frac{2497}{3628800}$ & $\frac{2497}{7257600}$ \\
2 & $-\frac{1}{2}$ & $\frac{11}{12}$ & $-\frac{3}{8}$ & $-\frac{19}{720}$ & $-\frac{11}{1440}$ & $-\frac{191}{60480}$ & $-\frac{191}{120960}$ & $-\frac{3233}{3628800}$ & $-\frac{7}{12800}$ \\
3 & $-\frac{1}{2}$ & $\frac{5}{12}$ & $\frac{1}{24}$ & $\frac{11}{720}$ & $\frac{11}{1440}$ & $\frac{271}{60480}$ & $\frac{13}{4480}$ & $\frac{7297}{3628800}$ & $\frac{425}{290304}$ \\
4 & $-\frac{1}{2}$ & $-\frac{1}{12}$ & $-\frac{1}{24}$ & $-\frac{19}{720}$ & $-\frac{3}{160}$ & $-\frac{863}{60480}$ & $-\frac{275}{24192}$ & $-\frac{33953}{3628800}$ & $-\frac{8183}{1036800}$ \\
5 & $\frac{1}{2}$ & $\frac{5}{12}$ & $\frac{3}{8}$ & $\frac{251}{720}$ & $\frac{95}{288}$ & $\frac{19087}{60480}$ & $\frac{5257}{17280}$ & $\frac{107017}{3628800}$ & $\frac{25713}{89600}$ \\
\hline
\end{tabular}

Analogously, the Gauss-Jackson corrector (52) can be written using a coefficient array $\alpha$

$$
\mathbf{r}_{\mathrm{n}}=h^{2}\left(\nabla^{-2} \ddot{\mathbf{r}}_{\mathrm{n}-1}+\sum_{i=0}^{N} \alpha_{\frac{N}{2}, i} \nabla^{i} \ddot{\mathbf{r}}_{\mathrm{n}}\right)
$$

The mid-correctors are then written

$$
\mathbf{r}_{\mathrm{n}}=h^{2}\left(\nabla^{-2} \ddot{\mathbf{r}}_{\mathrm{n}-1}+\sum_{i=0}^{N} \alpha_{n i} \nabla^{i} \ddot{\mathbf{r}}_{\frac{\mathrm{N}}{2}}\right)
$$

where $-\frac{N}{2} \leq n \leq \frac{N}{2}-1$. The coefficients of the mid-correctors are computed as the difference (54) of the corrector coefficients; the entire set of coefficients are thus computed recursively

$$
\alpha_{j i}= \begin{cases}\alpha_{j+1, i}-\alpha_{j+1, i-1} & \text { if } i>0 \\ \alpha_{j+1, i} & \text { if } i=0\end{cases}
$$

for $-\frac{N}{2} \leq j \leq \frac{N}{2}-1$. Finally the predictor is included in the array

$$
\mathbf{r}_{\mathrm{n}+1}=h^{2}\left(\nabla^{-2} \ddot{\mathbf{r}}_{\mathrm{n}}+\sum_{i=0}^{N} \alpha_{\frac{N}{2}+1, i} \nabla^{i} \ddot{\mathbf{r}}_{\mathrm{n}}\right)
$$

The eighth-order coefficients $\alpha_{j i}$ are presented in Table 4.

Note that the sum introduced by the $\nabla^{-2}$ term in the mid-correctors or corrector is accumulated through the point before the point being corrected; this is given by the $\ddot{\mathbf{r}}_{\mathrm{n}-1}$ term in (52). 
TABLE 4. Eighth-Order Gauss-Jackson Difference Coefficients, $\alpha_{j i}$

\begin{tabular}{|c|c|c|c|c|c|c|c|c|c|}
\hline \multirow[b]{2}{*}{$j$} & \multicolumn{9}{|c|}{$i$} \\
\hline & 0 & 1 & 2 & 3 & 4 & 5 & 6 & 7 & 8 \\
\hline-4 & $\frac{1}{12}$ & $-\frac{2}{3}$ & $\frac{559}{240}$ & $-\frac{371}{80}$ & $\frac{347539}{60480}$ & $-\frac{45601}{10080}$ & $\frac{7965611}{3628800}$ & $-\frac{427487}{725760}$ & $\frac{3250433}{53222400}$ \\
\hline-3 & $\frac{1}{12}$ & $-\frac{7}{12}$ & $\frac{419}{240}$ & $-\frac{347}{120}$ & $\frac{172651}{60480}$ & $-\frac{20191}{12096}$ & $\frac{1908311}{3628800}$ & $-\frac{8183}{129600}$ & $-\frac{330157}{159667200}$ \\
\hline-2 & $\frac{1}{12}$ & $-\frac{1}{2}$ & $\frac{299}{240}$ & $-\frac{79}{48}$ & $\frac{73111}{60480}$ & $-\frac{6961}{15120}$ & $\frac{33953}{518400}$ & $\frac{407}{172800}$ & $\frac{45911}{159667200}$ \\
\hline-1 & $\frac{1}{12}$ & $-\frac{5}{12}$ & $\frac{199}{240}$ & $-\frac{49}{60}$ & $\frac{23719}{60480}$ & $-\frac{275}{4032}$ & $-\frac{9829}{3628800}$ & $-\frac{641}{1814400}$ & $-\frac{3499}{53222400}$ \\
\hline 0 & $\frac{1}{12}$ & $-\frac{1}{3}$ & $\frac{119}{240}$ & $-\frac{77}{240}$ & $\frac{863}{12096}$ & $\frac{19}{6048}$ & $\frac{1571}{3628800}$ & $\frac{289}{3628800}$ & $\frac{317}{22809600}$ \\
\hline 1 & $\frac{1}{12}$ & $-\frac{1}{4}$ & $\frac{59}{240}$ & $-\frac{3}{40}$ & $-\frac{221}{60480}$ & $-\frac{31}{60480}$ & $-\frac{289}{3628800}$ & 0 & $\frac{317}{22809600}$ \\
\hline 2 & $\frac{1}{12}$ & $-\frac{1}{6}$ & $\frac{19}{240}$ & $\frac{1}{240}$ & $\frac{31}{60480}$ & 0 & $-\frac{289}{3628800}$ & $-\frac{289}{3628800}$ & $-\frac{3499}{53222400}$ \\
\hline 3 & $\frac{1}{12}$ & $-\frac{1}{12}$ & $-\frac{1}{240}$ & 0 & $\frac{31}{60480}$ & $\frac{31}{60480}$ & $\frac{1571}{3628800}$ & $\frac{641}{1814400}$ & $\frac{45911}{159667200}$ \\
\hline 4 & $\frac{1}{12}$ & 0 & $-\frac{1}{240}$ & $-\frac{1}{240}$ & $-\frac{221}{60480}$ & $-\frac{19}{6048}$ & $-\frac{9829}{3628800}$ & $-\frac{407}{172800}$ & $-\frac{330157}{159667200}$ \\
\hline 5 & $\frac{1}{12}$ & $\frac{1}{12}$ & $\frac{19}{240}$ & $\frac{3}{40}$ & $\frac{863}{12096}$ & $\frac{275}{4032}$ & $\frac{33953}{518400}$ & $\frac{8183}{129600}$ & $\frac{3250433}{53222400}$ \\
\hline
\end{tabular}

\section{Ordinate Forms}

The formulas given above all involve multiple differences and sums. In order to compute these formulas to a particular order, all the elements of the appropriate difference tables need to be computed. Calculating the differences can be timeconsuming and is unnecessary because the formulas can be re-expressed in terms of the function values themselves.

As shown above in equation (3), powers of the difference operators can be reduced to linear combinations of the original function values. The backward difference operator raised to any power can be expressed as a linear combination of the function value at the mesh points using binomial coefficients

$$
\nabla^{i} \ddot{\mathbf{r}}_{\mathrm{n}}=\sum_{m=0}^{i}\left(\begin{array}{c}
i \\
m
\end{array}\right)(-1)^{m} \ddot{\mathbf{r}}_{\mathrm{n}-\mathrm{m}}
$$

for $i \geq 0$. A sum over arbitrary coefficients $\zeta_{i}$ of the backward difference operator can thus be written in terms of the values of the function

$$
\begin{aligned}
\sum_{i=0}^{N} \zeta_{i} \nabla^{i} \dot{\mathbf{r}}_{\mathrm{n}} & =\sum_{\mathrm{i}=0}^{\mathrm{N}} \zeta_{i} \sum_{m=0}^{i}\left(\begin{array}{c}
i \\
m
\end{array}\right)(-1)^{m} \ddot{\mathbf{r}}_{\mathrm{n}-\mathrm{m}} \\
& =\sum_{m=0}^{N}(-1)^{m} \ddot{\mathbf{r}}_{\mathrm{n}-\mathrm{m}} \sum_{i=m}^{N} \zeta_{i}\left(\begin{array}{c}
i \\
m
\end{array}\right) \\
& =\sum_{m=0}^{N} z_{N m} \ddot{\mathbf{r}}_{\mathrm{n}-\mathrm{m}}
\end{aligned}
$$


with the ordinate coefficients defined as

$$
z_{N m}=(-1)^{m} \sum_{i=m}^{N} \zeta_{i}\left(\begin{array}{c}
i \\
m
\end{array}\right)
$$

Notice that these coefficients, unlike the difference coefficients $\zeta$, depend on $N$, the order of the integration method. Thus a variation of order to control errors while integrating is feasible in difference form, but is more difficult in ordinate form. Here the index $m$ numbers the backpoints so the current point is $m=0$ and each previous point has a higher positive value of $m$.

As an example, consider the summed Adams corrector (38). For a fourth-order method, $N=4$, the difference coefficients $\zeta_{i}$ and the ordinate coefficients $z_{4 m}$ are

\begin{tabular}{c|ccccc}
$m$ & 0 & 1 & 2 & 3 & 4 \\
\hline$\zeta_{i}$ & $-\frac{1}{2}$ & $-\frac{1}{12}$ & $-\frac{1}{24}$ & $-\frac{19}{720}$ & $-\frac{3}{160}$ \\
$z_{4 m}$ & $-\frac{193}{288}$ & $\frac{77}{240}$ & $-\frac{7}{30}$ & $\frac{73}{720}$ & $-\frac{3}{160}$
\end{tabular}

An alternative formulation includes the $\nabla^{0}$ term $-\frac{1}{2} \ddot{\mathbf{r}}_{\mathrm{n}}$ with the sum rather than with the coefficients. The example summed Adams-Moulton corrector (38) is now written

$$
\dot{\mathbf{r}}_{\mathrm{n}}=h\left[\nabla^{-1} \ddot{\mathbf{r}}_{\mathrm{n}}-\frac{1}{2} \ddot{\mathbf{r}}_{\mathrm{n}}+\left(-\frac{1}{12} \nabla-\frac{1}{24} \nabla^{2}-\frac{19}{720} \nabla^{3}-\frac{3}{160} \nabla^{4}+\ldots\right) \ddot{\mathbf{r}}_{\mathrm{n}}\right]
$$

With this formulation the $m=0$ coefficients are different from (68)

\begin{tabular}{c|ccccc}
$m$ & 0 & 1 & 2 & 3 & 4 \\
\hline$\zeta_{i}$ & 0 & $-\frac{1}{12}$ & $-\frac{1}{24}$ & $-\frac{19}{720}$ & $-\frac{3}{160}$ \\
$z_{4 m}$ & $-\frac{49}{288}$ & $\frac{77}{240}$ & $-\frac{7}{30}$ & $\frac{73}{720}$ & $-\frac{3}{160}$
\end{tabular}

All the single-integration corrector and mid-corrector coefficients are computed this way; however, the $\nabla^{0}$ term is included in the coefficients for the predictor. The eighth-order summed Adams coefficients in ordinate form $b_{j k}$ are shown in Table 5, and the Gauss-Jackson coefficients $a_{j k}$ are shown in Table 6. For Gauss-Jackson, the $\nabla^{0}$ term $\frac{1}{12} \ddot{\mathbf{r}}_{\mathrm{n}}$, is included with the coefficients and not the sum $\nabla^{-2}$. Although this alternate formulation is not necessary, it makes the programming easier, as described in the next section. Both the Gauss-Jackson and the summed Adams coefficients may be compared with [6], Table III (pages 25-26) and [12] Appendix C.3. Ordinate forms for the Adams, summed Adams, Störmer-Cowell, and Gauss-Jackson predictors and correctors for orders between 1 and 14 may also be found in [5].

In this section, the $\zeta$ are generic difference coefficients such as $\alpha$ and $\beta$, and the $z$ are the corresponding ordinate coefficients, which have an extra subscript to indicate the order. The actual eighth-order ordinate coefficients $a$ or $b$ do not have an order subscript even though they are dependent on the order; the two subscripts are the point of integration $j$ and the point of reference $k$. 


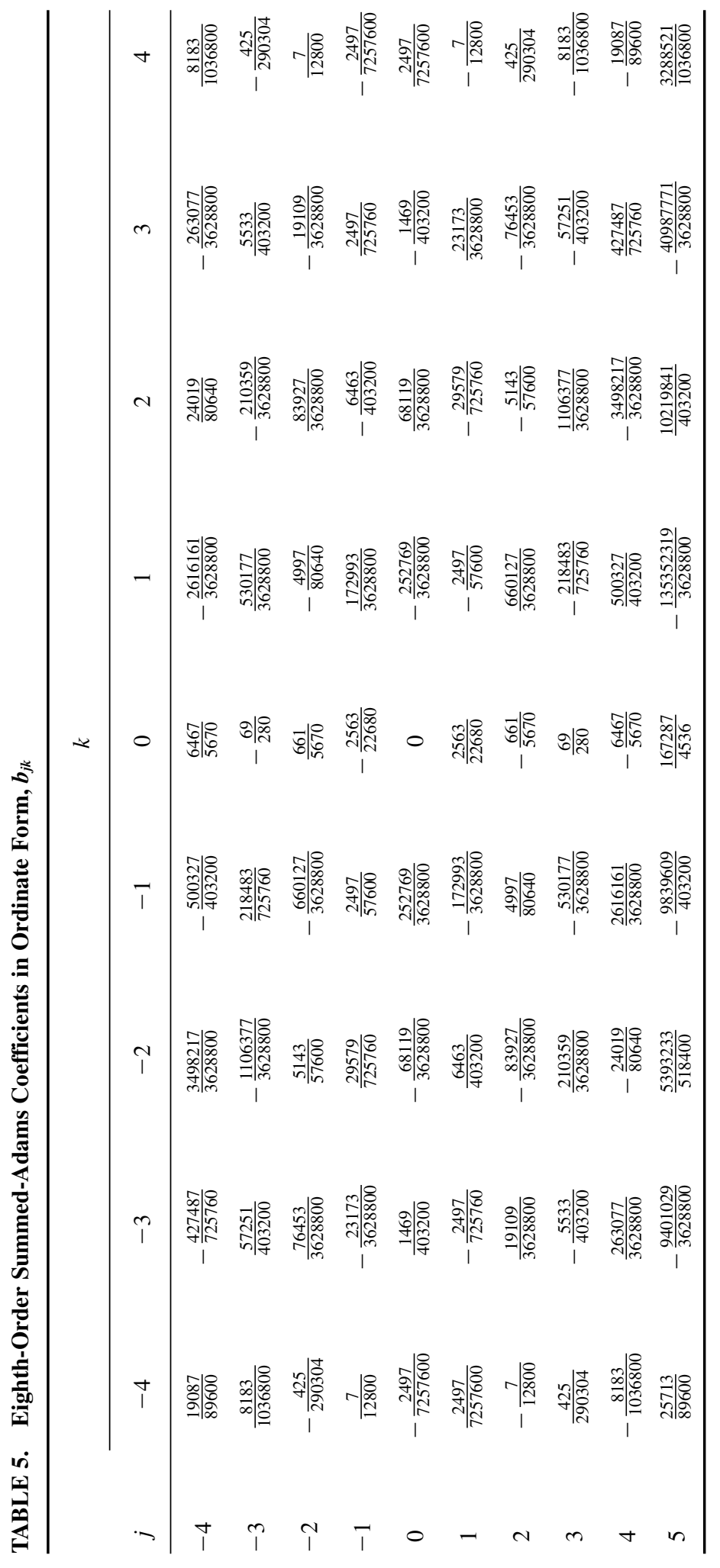




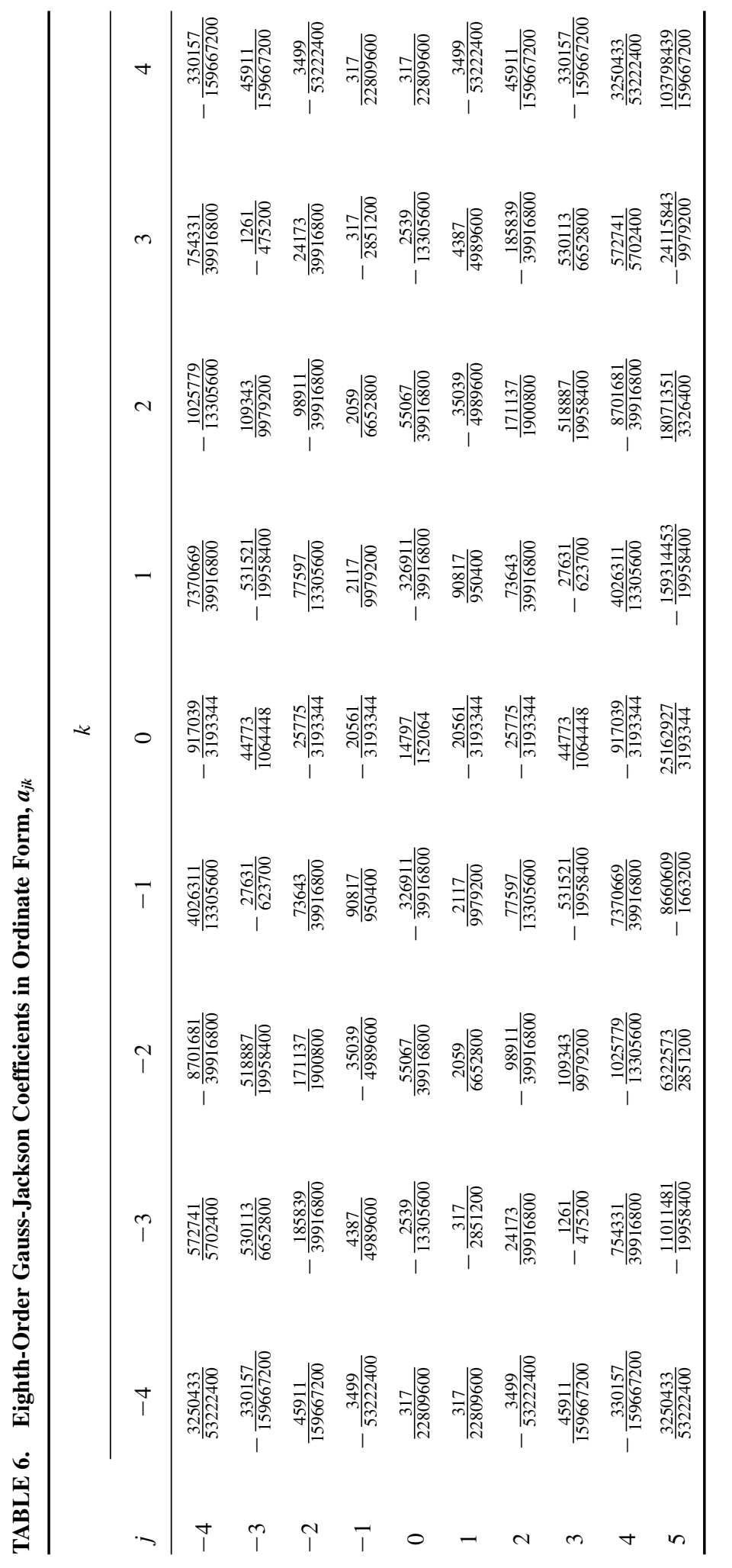




\section{Implementation}

To find both position and velocity given a function for acceleration, either a single-integration method must be used once for velocity and the method used again for position, or a single-integration method must be used along with a double-integration method. Assuming that summed and nonsummed forms are not used together, the four integrators derived above offer four implementations: two Adams, two summed Adams, Adams and Störmer-Cowell, or summed Adams and Gauss-Jackson integration. For convenience, Gauss-Jackson integration is understood to mean Gauss-Jackson and summed Adams integration, and Störmer-Cowell integration is understood to mean Störmer-Cowell and Adams integration. Because Gauss-Jackson integration involves sum terms, its implementation is somewhat complicated, and so is described in detail in this section.

\section{Overview}

In order to use the predictor-corrector formulas to integrate forward in time with an $N^{\text {th }}$ order method, $N+1$ points must already be known. To preserve the order of the method, these $N+1$ points must have also been found using an $N^{\text {th }}$ order method. Since initially only one point, epoch, is known, a startup procedure is necessary. If $N+1$ initial estimated points are given, the mid-corrector and corrector formulas can be used to refine them. By applying the mid-correctors iteratively until the points converge, the resulting points are accurate through $N^{\text {th }}$ order.

For the eighth-order methods used in this study, a Taylor expansion of the twobody solution to fifth order ( $f$ and $g$ series, [14] equation (4-68)) is used for the initial estimate, though a single-step integrator, such as Runge-Kutta could be used. The analytic solution generates eight positions and velocities in addition to epoch: four before and four after epoch. The accelerations at all nine points are then found from the positions and velocities with full perturbations, and corrector and midcorrector formulas are applied to the eight non-epoch accelerations, which generates corrected positions and velocities. These corrected positions and velocities are used to find corrected accelerations, and the cycle repeats, until the accelerations converge, i.e., on two successive iterations, the absolute difference is less than a specified tolerance. This process is denoted SECECE...CE, where "S" is startup estimate, " $\mathrm{E}$ " is evaluate, and "C" is correct. This process is done for the eight initial points other than epoch.

Once the startup points have been corrected to satisfactory accuracy, the regular predictor-corrector process can start. First the predictor equation is used to find the position and velocity of the first point after startup, and the force model is evaluated to find the acceleration of that point. This predicted acceleration is then used in the corrector formula to find a corrected position and velocity. The corrected position and velocity may be compared to the predicted values, and if they do not match to some tolerance, another acceleration is evaluated, which is then used in the corrector. Note that the procedure is different from startup, where convergence of acceleration is checked. This cycle would then be repeated until the position and velocity converge, or until some specified maximum number of iterations is reached. This process gives a $\mathrm{P}(\mathrm{EC})^{n}$ implementation, with a maximum value of $n$ specified.

\section{Single Integration}

The first step in startup of single integration is to use the mid-corrector formulas to correct the eight estimated velocities around epoch. In difference form, these 
formulas are (58); using the ordinate coefficients $b$, the formulas are

$$
\dot{\mathbf{r}}_{\mathrm{n}}=h\left(\nabla^{-1} \ddot{\mathbf{r}}_{\mathrm{n}}-\frac{\ddot{\mathbf{r}}_{\mathrm{n}}}{2}+\sum_{k=-4}^{4} b_{n k} \ddot{\mathbf{r}}_{\mathrm{k}}\right)
$$

with $-4 \leq n \leq 4$. Note the term $-\frac{\ddot{r}_{n}}{2}$ is excluded from the coefficient sum in anticipation of its inclusion in the acceleration term, as in (69). The integration constant $\mathbf{C}_{1}$ (see Tables and Operators) may be determined by making sure that the initial conditions $(n=0)$

$$
\dot{\mathbf{r}}_{0}=h\left(\nabla^{-1} \ddot{\mathbf{r}}_{0}-\frac{\ddot{\mathbf{r}}_{0}}{2}+\sum_{k=-4}^{4} b_{0 k} \ddot{\mathbf{r}}_{\mathrm{k}}\right)
$$

are satisfied; solving for $\mathbf{C}_{1}=\nabla^{-1} \ddot{\mathbf{r}}_{0}$

$$
\mathbf{C}_{1}=\frac{\dot{\mathbf{r}}_{0}}{h}-\sum_{k=-4}^{4} b_{0 k} \ddot{\mathbf{r}}_{\mathrm{k}}+\frac{\ddot{\mathbf{r}}_{0}}{2}
$$

The acceleration term may be included with the sum to make software implementation easier. Define the term $\mathbf{s}_{\mathrm{n}}=\nabla^{-1} \ddot{\mathbf{r}}_{\mathrm{n}}-\frac{1}{2} \ddot{\mathbf{r}}_{\mathrm{n}}$, which replaces the first two terms in (71), and define a new integration constant $\mathbf{C}_{1}^{\prime}=\mathbf{C}_{1}-\frac{\ddot{\mathbf{r}}_{0}}{2}$. Then (71) may be rewritten

$$
\dot{\mathbf{r}}_{\mathrm{n}}=h\left(\mathbf{s}_{\mathrm{n}}+\sum_{k=-4}^{4} b_{n k} \ddot{\mathbf{r}}_{\mathrm{k}}\right)
$$

where $n$ ranges from -4 to 4 , with

$$
\mathbf{s}_{\mathrm{n}}= \begin{cases}\mathbf{s}_{\mathrm{n}-1}+\frac{\ddot{\mathbf{r}}_{\mathrm{n}-1}+\ddot{\mathbf{r}}_{\mathrm{n}}}{2} & \text { if } n>0 \\ \mathbf{C}_{1}^{\prime} & \text { if } n=0 \\ \mathbf{s}_{\mathrm{n}+1}-\frac{\ddot{\mathbf{r}}_{\mathrm{n}+1}+\ddot{\mathbf{r}}_{\mathrm{n}}}{2} & \text { if } n<0\end{cases}
$$

Table 7 shows the relationship between the summed accelerations and $\mathbf{s}_{\mathrm{n}}$ near epoch. For a given value of $n$, notice the symmetry of the formulas in the last column; changing the sign of $n$ merely changes the sign of the term added to $\mathbf{C}_{1}^{\prime}$. This formulation makes programming simpler and is the reason that the term $-\ddot{\mathbf{r}}_{0} / 2$ is moved from the coefficients to the sum. The $\mathbf{s}_{\mathrm{n}}$ are computed successively in order to compute the startup velocities. While the formulas are correct for $n=0$, no correction is performed on epoch.

TABLE 7. Relationship Between Summation Term and $s_{n}$

\begin{tabular}{lccc}
\hline$n$ & $\ddot{\mathbf{r}}_{\mathrm{n}}$ & $\nabla^{-1} \ddot{\mathbf{r}}_{\mathrm{n}}$ & $\mathbf{S}_{\mathrm{n}}=\nabla^{-1} \ddot{\mathbf{r}}_{\mathrm{n}}-\frac{1}{2} \ddot{\mathbf{r}}_{\mathrm{n}}$ \\
\hline-2 & $\ddot{\mathbf{r}}_{-2}$ & $\mathbf{C}_{1}-\ddot{\mathbf{r}}_{0}-\ddot{\mathbf{r}}_{-1}$ & $\mathbf{C}_{1}^{\prime}-\frac{1}{2} \ddot{\mathbf{r}}_{0}-\ddot{\mathbf{r}}_{-1}-\frac{1}{2} \ddot{\mathbf{r}}_{-2}$ \\
-1 & $\ddot{\mathbf{r}}_{-1}$ & $\mathbf{C}_{1}-\ddot{\mathbf{r}}_{0}$ & $\mathbf{C}_{1}^{\prime}-\frac{1}{2} \ddot{\mathbf{r}}_{0}-\frac{1}{2} \ddot{\mathbf{r}}_{-1}$ \\
0 & $\ddot{\mathbf{r}}_{0}$ & $\mathbf{C}_{1}$ & $\mathbf{C}_{1}^{\prime}$ \\
1 & $\ddot{\mathbf{r}}_{1}$ & $\mathbf{C}_{1}+\ddot{\mathbf{r}}_{1}$ & $\mathbf{C}_{1}^{\prime}+\frac{1}{2} \ddot{\mathbf{r}}_{0}+\frac{1}{2} \ddot{\mathbf{r}}_{1}$ \\
2 & $\ddot{\mathbf{r}}_{2}$ & $\mathbf{C}_{1}+\ddot{\mathbf{r}}_{1}+\ddot{\mathbf{r}}_{2}$ & $\mathbf{C}_{1}^{\prime}+\frac{1}{2} \ddot{\mathbf{r}}_{0}+\ddot{\mathbf{r}}_{1}+\frac{1}{2} \ddot{\mathbf{r}}_{2}$ \\
\hline
\end{tabular}


Once the startup points have been corrected, integration may proceed forward with application of the predictor, followed by one or more applications of the corrector. The predictor finds the velocity $\dot{\mathbf{r}}_{\mathrm{n}+1}$ from the coefficients in row 5 of the $b$ array, and the nine most recent accelerations $\ddot{\mathbf{r}}_{\mathrm{n}-8} \ldots \ddot{\mathbf{r}}_{\mathrm{n}}$. The formula also includes a $\nabla^{-1}$ term which is the sum of all the accelerations since epoch and $\mathbf{C}_{1}$

$$
\dot{\mathbf{r}}_{\mathrm{n}+1}=h\left(\nabla^{-1} \ddot{\mathbf{r}}_{\mathrm{n}}+\sum_{k=-4}^{4} b_{5 k} \ddot{\mathbf{r}}_{\mathrm{n}+\mathrm{k}-4}\right)
$$

with $n \geq 4$. This expression differs from the difference form given in (60). For programming, the predictor can be written using $\mathbf{s}_{\mathrm{n}}$

$$
\dot{\mathbf{r}}_{\mathrm{n}+1}=h\left(\mathbf{s}_{\mathrm{n}}+\frac{\ddot{\mathbf{r}}_{\mathrm{n}}}{2}+\sum_{k=-4}^{4} b_{5 k} \dot{\mathbf{r}}_{\mathrm{n}+\mathrm{k}-4}\right)
$$

The Adams-Moulton corrector formula in difference form (69), for any point $n>4$ after startup, can be rewritten using the eighth-order ordinate coefficients $b$. These coefficients are applied to the eight previous accelerations and the current predicted acceleration

$$
\dot{\mathbf{r}}_{\mathrm{n}}=h\left(\nabla^{-1} \ddot{\mathbf{r}}_{\mathrm{n}}-\frac{\ddot{\mathbf{r}}_{\mathrm{n}}}{2}+\sum_{k=-4}^{4} b_{4 k} \ddot{\mathbf{r}}_{\mathrm{n}+\mathrm{k}-4}\right)
$$

with $n \geq 4$. Note that (78) is the same as (71) for $n=4$. The corrector can be written using $\mathbf{S}_{\mathrm{n}}$

$$
\dot{\mathbf{r}}_{\mathrm{n}}=h\left(\mathbf{s}_{\mathrm{n}}+\sum_{k=-4}^{4} b_{4 k} \ddot{\mathbf{r}}_{\mathrm{n}+\mathrm{k}-4}\right)
$$

Depending on the implementation, this equation may be applied through several evaluate and correct (EC) iterations, but only the last acceleration, $k=4$, changes, so the summation for the first eight need only be done once, then saved through the iterations.

Note that when applying the predictor, the first formula in (75) uses one half of the corrected acceleration and one half of the predicted acceleration in the sum. If the acceleration term is not shifted, then the entire acceleration comes from the predicted value. Therefore, the shift has a presumed advantage of using half the corrected acceleration and half the predicted, rather than all predicted acceleration. A possible further improvement would be to use $\nabla^{-1} \dot{\mathbf{r}}_{\mathrm{n}-1}$ instead of $\nabla^{-1} \dot{\mathbf{r}}_{\mathrm{n}}$ in (78) with $b$ redefined appropriately. Then this summation would have only corrected values.

\section{Double Integration}

The mid-correctors, predictor, and corrector for the second integration ordinate form are respectively

$$
\begin{array}{rlrl}
\mathbf{r}_{\mathrm{n}} & =h^{2}\left(\nabla^{-2} \ddot{\mathbf{r}}_{\mathrm{n}-1}+\sum_{k=-4}^{4} a_{n k} \ddot{\mathbf{r}}_{\mathrm{k}}\right) & -4 \leq n \leq 4 \\
\mathbf{r}_{\mathrm{n}+1}=h^{2}\left(\nabla^{-2} \ddot{\mathbf{r}}_{\mathrm{n}}+\sum_{k=-4}^{4} a_{5 k} \ddot{\mathbf{r}}_{\mathrm{n}+\mathrm{k}-4}\right) & n \geq 4 \\
\mathbf{r}_{\mathrm{n}}=h^{2}\left(\nabla^{-2} \ddot{\mathbf{r}}_{\mathrm{n}-1}+\sum_{k=-4}^{4} a_{4 k} \ddot{\mathbf{r}}_{\mathrm{n}+\mathrm{k}-4}\right) & n \geq 4
\end{array}
$$


corresponding to the difference form (62), (64), (61). To find the integration constant for double integration, $\mathbf{C}_{2}$, use $n=0$ in (80)

$$
\mathbf{r}_{0}=h^{2}\left(\nabla^{-2} \ddot{\mathbf{r}}_{-1}+\sum_{k=-4}^{4} a_{0 k} \ddot{\mathbf{r}}_{\mathrm{k}}\right)
$$

Table 2 shows that the second sum on the point immediately before epoch is the difference of the two constants of integration

$$
\nabla^{-2} \ddot{\mathbf{r}}_{-1}=\mathbf{C}_{2}-\mathbf{C}_{1}
$$

which than allows us to solve for $\mathbf{C}_{2}$, because $\mathbf{C}_{1}$ is known (73)

$$
\mathbf{C}_{2}=\frac{\mathbf{r}_{0}}{h^{2}}-\sum_{k=-4}^{4} a_{0 k} \ddot{\mathbf{r}}_{\mathrm{k}}+\mathbf{C}_{1}
$$

For software implementation, a term $\mathbf{S}_{\mathrm{n}}=\nabla^{-2} \ddot{\mathbf{r}}_{\mathrm{n}-1}$ is defined recursively

$$
\mathbf{S}_{\mathrm{n}}= \begin{cases}\mathbf{S}_{\mathrm{n}-1}+\mathbf{S}_{\mathrm{n}-1}+\frac{\ddot{\mathbf{r}}_{\mathrm{n}-1}}{2} & \text { if } n>0 \\ \mathbf{C}_{2}-\mathbf{C}_{1} & \text { if } n=0 \\ \mathbf{S}_{\mathrm{n}+1}-\mathbf{S}_{\mathrm{n}+1}+\frac{\ddot{\mathbf{r}}_{\mathrm{n}+1}}{2} & \text { if } n<0\end{cases}
$$

The mid-correctors, predictor, and corrector can now be written in terms of $\mathbf{S}_{\mathrm{n}}$

$$
\begin{array}{rlrl}
\mathbf{r}_{\mathrm{n}} & =h^{2}\left(\mathbf{S}_{\mathrm{n}}+\sum_{k=-4}^{4} a_{n k} \ddot{\mathbf{r}}_{\mathrm{k}}\right) & -4 \leq n \leq 4 \\
\mathbf{r}_{\mathrm{n}+1}=h^{2}\left(\mathbf{S}_{\mathrm{n}+1}+\sum_{k=-4}^{4} a_{5 k} \ddot{\mathbf{r}}_{\mathrm{n}+\mathrm{k}-4}\right) & n \geq 4 \\
\mathbf{r}_{\mathrm{n}}=h^{2}\left(\mathbf{S}_{\mathrm{n}}+\sum_{k=-4}^{4} a_{4 k} \ddot{\mathbf{r}}_{\mathrm{n}+\mathrm{k}-4}\right) & n \geq 4
\end{array}
$$

As with first integration, the summation in the corrector may be split, because the first eight accelerations do not change during the EC iteration.

Procedure

A step-by-step procedure for the operation of the integrator can now be written:

\section{$\underline{\text { Startup }}$}

1. Use $f$ and $g$ series to calculate eight positions and velocities surrounding epoch labelled $\mathbf{r}_{-4} \ldots \mathbf{r}_{4}$ with $\mathbf{r}_{0}$ epoch.

2. Evaluate nine accelerations from these positions and velocities, and those of epoch labelled $\ddot{\mathbf{r}}_{-4} \ldots \ddot{\mathbf{r}}_{4}$.

3. While the accelerations have not converged:

(a) Calculate $\mathbf{s}_{0}$ (75) and $\mathbf{S}_{0}$ (86).

(b) For each point $n=-4 \ldots 4, n \neq 0$ :

i. Calculate $\mathbf{S}_{\mathrm{n}}(75)$ and $\mathbf{S}_{\mathrm{n}}(86)$.

ii. Calculate $\sum_{k=-4}^{4} b_{n k} \ddot{\mathbf{r}}_{\mathrm{k}}$ using Table 5 .

iii. Calculate $\sum_{k=-4}^{4} a_{n k} \ddot{\mathbf{r}}_{\mathrm{k}}$ using Table 6 . 
iv. Calculate $\dot{\mathbf{r}}_{\mathrm{n}}(74)$ and $\mathbf{r}_{\mathrm{n}}(87)$.

v. Evaluate the updated acceleration $\ddot{\mathbf{r}}_{\mathrm{n}}$ using the appropriate force model. (c) Test convergence of the accelerations.

Predict

4. Calculate $\mathbf{S}_{\mathrm{n}+1}$ (86).

5. Calculate $\sum_{k=-4}^{4} b_{5 k} \ddot{\mathbf{r}}_{\mathrm{n}+\mathrm{k}-4}$.

6. Calculate $\sum_{k=-4}^{4} a_{5 k} \dot{\mathbf{r}}_{\mathrm{n}+\mathrm{k}-4}$.

7. Calculate $\dot{\mathbf{r}}_{\mathrm{n}+1}$ (77) and $\mathbf{r}_{\mathrm{n}+1}$ (88).

Evaluate- Correct

8. Evaluate the acceleration $\ddot{\mathbf{r}}_{\mathrm{n}+1}$.

9. Increment $n$.

10. While $\dot{\mathbf{r}}_{\mathrm{n}}$ and $\mathbf{r}_{\mathrm{n}}$ have not converged and the maximum number of corrector iterations is not exceeded:

(a) Calculate $\mathbf{s}_{\mathrm{n}}$ (75).

(b) If first iteration:

i. Calculate $\sum_{k=-4}^{3} b_{4 k} \ddot{\mathbf{r}}_{\mathrm{n}+\mathrm{k}-4}$.

ii. Calculate $\sum_{k=-4}^{3} a_{4 k} \ddot{\mathbf{r}}_{\mathrm{n}+\mathrm{k}-4}$.

(c) Calculate $b_{4 k} \ddot{\mathbf{r}}_{\mathrm{n}}$ and $a_{4 k} \ddot{\mathbf{r}}_{\mathrm{n}}$.

(d) Sum the results of 10(a), 10(b), and 10(c) to obtain $\dot{\mathbf{r}}_{\mathrm{n}}$ (79) and $\mathbf{r}_{\mathrm{n}}$ (89).

(e) Test convergence of $\dot{\mathbf{r}}_{\mathrm{n}}$ and $\mathbf{r}_{\mathrm{n}}$; if not converged, and this is not the last allowable corrector iteration, evaluate $\ddot{\mathbf{r}}_{\mathrm{n}}$.

11. Go to Step 4 unless this is the final time desired.

Similar procedures are used for implementing two Adams, two summed Adams, or Adams with Störmer-Cowell.

\section{Effects of Step Size and Order on Accuracy and Stability}

In general, the accuracy of a numerical integrator improves as the step size is reduced and the order of the method is increased. One exception to this rule is that round-off error can occur if the step size becomes too small. Another exception occurs when the integrator becomes unstable. Increasing the order may cause instability because higher-order methods are susceptible to instability at large step sizes [15] (pp. 139, 146).

To assess the effect of step size and order on accuracy, we compare ephemeris generated using step sizes of 30,60, 120, and 240 seconds, with 6th, 8th, 10th, 12th, and 14th order Gauss-Jackson integrators. The integrators follow the procedure given in the Procedure section, modified for the order of the integrator. The tests compare ephemeris generated at one minute intervals over 72 hours. For step sizes greater than one minute, a fifth-order Hermitian interpolator ([16], p. 28) is used to find the intermediate points. The perturbation forces include $24 \times 24$ WGS-84 geopotential [17], Jacchia 70 [18] drag, and lunar and solar forces. Note that all of these perturbations are continuous forces. To simplify this study, discontinuous forces such as solar radiation pressure are not considered. The two test cases used are the International Space Station (ISS, catalog number 25544), with a period 
of $92.05 \mathrm{~min}$. and eccentricity of 0.001, and CRRES (20712), with a period of $607.28 \mathrm{~min}$. and eccentricity of 0.716 .

In the tests, a metric for integration accuracy is defined using an error ratio defined in terms of the RMS error of the integration [19]. First define position errors as

$$
\Delta r=\left|r_{\text {computed }}-r_{\text {reference }}\right|
$$

The RMS position error can be calculated

$$
\Delta r_{\mathrm{RMS}}=\sqrt{\frac{1}{N} \sum_{i=1}^{N}\left(\Delta r_{i}\right)^{2}}
$$

The RMS position error is normalized by the apogee distance and the number of orbits to find the position error ratio

$$
\rho_{r}=\frac{\Delta r_{\mathrm{RMS}}}{r_{A} N_{\text {orbits }}}
$$

The error ratios for ISS are given in Table 8, and the error ratios for CRRES are given in Table 9. In the comparison the ephemeris generated by the 14th order method with 30 second step size is considered to be the reference. The tables show that step size affects accuracy more than the order of the method does. The trend is similar for both the circular and the eccentric objects. In Table 8 the asterisk denotes that the integration has become unstable. It is obvious when instability has occurred because the eccentricity of the integrated ephemeris starts to grow to the point of the orbit becoming hyperbolic. The instability at a large step size for high orders shows that if computation time is a higher priority than accuracy, a lower order method is preferable, because a larger step size can be used without the threat of instability.

Herrick [10] claims that the corrector is not usually required in Gauss-Jackson integration. This saves computation time, because only one evaluation is required (PE) per step. A predictor-only form of Gauss-Jackson can be created by skipping steps $10(\mathrm{~b})-10(\mathrm{e})$ of the procedure above. Tables 10 and 11 list error ratios for predictor-only results for ISS and CRRES, respectively. Once again, the 14th order, 30 second step size results, with the corrector, are considered to be the reference. Comparing Table 10 to Table 8 , we see that for the circular satellite the results with and without the corrector are similar for smaller step sizes and lower orders.

TABLE 8. Error Ratios for ISS $(e=\mathbf{0 . 0 0 1})$

\begin{tabular}{cccccc}
\hline & \multicolumn{5}{c}{ Order } \\
\cline { 2 - 5 } $\begin{array}{c}\text { Step Size } \\
(\mathrm{sec})\end{array}$ & 6 & 8 & 10 & 12 & 14 \\
\hline 30 & $1.0 \times 10^{-11}$ & $1.5 \times 10^{-12}$ & $3.8 \times 10^{-13}$ & $1.5 \times 10^{-13}$ & - \\
60 & $2.4 \times 10^{-9}$ & $1.5 \times 10^{-9}$ & $1.1 \times 10^{-9}$ & $9.7 \times 10^{-10}$ & $9.0 \times 10^{-10}$ \\
120 & $9.7 \times 10^{-8}$ & $1.1 \times 10^{-7}$ & $1.1 \times 10^{-7}$ & $8.8 \times 10^{-8}$ & $1.1 \times 10^{-7}$ \\
240 & $1.1 \times 10^{-4}$ & $1.3 \times 10^{-4}$ & $1.2 \times 10^{-4}$ & $*$ & $*$ \\
\hline
\end{tabular}


TABLE 9. Error Ratios for CRRES $(e=0.716)$

\begin{tabular}{cccccc}
\hline & \multicolumn{5}{c}{ Order } \\
\cline { 2 - 5 } $\begin{array}{c}\text { Step Size } \\
\text { (sec) }\end{array}$ & 6 & 8 & 10 & 12 & 14 \\
\hline 30 & $1.4 \times 10^{-11}$ & $2.5 \times 10^{-13}$ & $9.4 \times 10^{-14}$ & $1.1 \times 10^{-14}$ & - \\
60 & $5.3 \times 10^{-9}$ & $3.9 \times 10^{-11}$ & $1.2 \times 10^{-10}$ & $1.6 \times 10^{-10}$ & $1.6 \times 10^{-10}$ \\
120 & $1.1 \times 10^{-7}$ & $7.6 \times 10^{-7}$ & $2.2 \times 10^{-7}$ & $2.6 \times 10^{-8}$ & $8.7 \times 10^{-8}$ \\
240 & $9.3 \times 10^{-4}$ & $1.9 \times 10^{-5}$ & $4.0 \times 10^{-4}$ & $2.0 \times 10^{-4}$ & $9.0 \times 10^{-5}$ \\
\hline
\end{tabular}

However, when the corrector is not used instability occurs at lower orders and smaller step sizes than when the corrector is used. For the eccentric satellite, shown in Table 11, there is a somewhat more noticeable loss in accuracy when the corrector is not applied. However, the integration of the eccentric satellite remains stable in more cases than the circular case.

In general, when the corrector is applied at least two evaluations are performed (PECE). This means that a predictor-only implementation (PE) has roughly half the computation time as a predictor-corrector implementation, because evaluations account for most of the computation time when using a realistic force model. Computation time is also related to the step size; when the step size is doubled the computation time is halved. Comparing Table 8 to Table 10, and Table 9 to Table 11, we see that 30 second predictor-only results are more accurate than 60 second predictor-corrector results, though these have roughly the same computation time. This justifies Herrick's claim that the corrector is not necessary; however, if it is not used, care must be taken to avoid instability. The inclusion of the corrector (PEC) with no final evaluation gives an improvement in accuracy over the predictor-only method, with little cost in computation time, since there are no extra evaluations. However this method has the same stability problems as the predictor-only method.

An alternate method performs a second evaluation in which only the two-body force is re-evaluated; the perturbation force from the first evaluation is re-used. Because the two-body evaluation is a simple calculation, this method has essentially the same run-time as performing only one evaluation per step. However, in most

TABLE 10. Error Ratios for ISS, Predictor only $(e=0.001)$

\begin{tabular}{cccccc}
\hline & \multicolumn{5}{c}{ Order } \\
\cline { 2 - 5 } $\begin{array}{c}\text { Step Size } \\
(\mathrm{sec})\end{array}$ & 6 & 8 & 10 & 12 & 14 \\
\hline 30 & $1.4 \times 10^{-11}$ & $1.9 \times 10^{-12}$ & $3.5 \times 10^{-13}$ & $1.5 \times 10^{-12}$ & $*$ \\
60 & $4.7 \times 10^{-9}$ & $1.6 \times 10^{-9}$ & $9.2 \times 10^{-10}$ & $*$ & $*$ \\
120 & $1.3 \times 10^{-6}$ & $1.2 \times 10^{-7}$ & $*$ & $*$ & $*$ \\
240 & $4.9 \times 10^{-4}$ & $*$ & $*$ & $*$ & $*$ \\
\hline
\end{tabular}


TABLE 11. Error Ratios for CRRES, Predictor only $(e=0.716)$

\begin{tabular}{|c|c|c|c|c|c|}
\hline \multirow{2}{*}{$\begin{array}{l}\text { Step Size } \\
(\mathrm{sec})\end{array}$} & \multicolumn{5}{|c|}{ Order } \\
\hline & 6 & 8 & 10 & 12 & 14 \\
\hline 30 & $3.5 \times 10^{-10}$ & $6.6 \times 10^{-12}$ & $1.8 \times 10^{-13}$ & $2.1 \times 10^{-13}$ & $5.4 \times 10^{-13}$ \\
\hline 60 & $8.8 \times 10^{-8}$ & $2.0 \times 10^{-9}$ & $1.9 \times 10^{-9}$ & $1.4 \times 10^{-9}$ & $5.8 \times 10^{-7}$ \\
\hline 120 & $4.2 \times 10^{-5}$ & $2.3 \times 10^{-5}$ & $2.0 \times 10^{-6}$ & $4.3 \times 10^{-6}$ & $*$ \\
\hline 240 & $1.3 \times 10^{-2}$ & $1.0 \times 10^{-2}$ & $1.4 \times 10^{-3}$ & $*$ & $*$ \\
\hline
\end{tabular}

cases this method has the same stability advantage as performing two full evaluations, though the partial evaluation is not as accurate as a full evaluation. Results using a partial evaluation are available in [9].

\section{Summary}

This paper derives the Gauss-Jackson integration method widely used in special perturbations software for space surveillance. Both single and double integration are derived from difference tables. The ordinate form simplifies the calculations because it avoids the need to calculate tables of differences; however, it requires new sets of coefficients. We show how the theory is actually implemented in the software. Finally, there is a discussion of stability and accuracy which shows that step size has more of an effect on accuracy than the order of the method does, and that higher orders are subject to instability at large step sizes.

Coefficients may be computed to any order with code that is available at the permanent link http://hdl.handle.net/1903/2202.

\section{Acknowledgments}

We thank Keith Atkins, Shannon Coffey, Felix Hoots, and Alan Segerman for helpful comments on the original paper. Fred Lutze provided comments on this version of the paper, and we dedicate it to his memory.

\section{References}

[1] JACKSON, J. "Note on the Numerical Integration of $\mathrm{d}^{2} x / \mathrm{d} t^{2}=f(x, t)$," Monthly Notes, Volume 84, Royal Astronomy Society, 1924, pp. 602-606.

[2] KOSKELA, P.E. "Astrodynamic Analysis for the Advanced Orbit/Ephemeris Subsystem," Technical Report U-4180, Philco-Ford Corporation, Aeronutronic Division, Newport Beach, California, September 1967.

[3] NEAL, H. L., COFFEY, S. L., and KNOWLES, S. "Maintaining the Space Object Catalog with Special Perturbations," in F. Hoots, B. Kaufman, P. Cefola, and D. Spencer, editors, Astrodynamics 1997 Part II, Volume 97 of Advances in the Astronautical Sciences, pp. 1349-1360.

[4] CASALI, S. "Conjunction Analysis Approach for the Astrodynamics Support Workstation (ASW)," presented at the Core Technologies for Space Systems Conference, Colorado Springs, Colorado, November 19-21, 2002.

[5] MAURY, J. L. and SEGAL, G. P. "Cowell Type Numerical Integration as Applied to Satellite Orbit Computation,” Technical Report X-553-69-46, NASA, 1969, NTIS \#N6926703. 
[6] NORAD "Mathematical Foundation for SCC Astrodynamic Theory," Technical Report TP SCC 008, Headquarters North American Aerospace Defense Command, 1982. Obtainable from Defense Technical Information Center, Cameron Station, Alexandria, VA 22304-6145, as AD \#B081394.

[7] WOODBURN, J. "Mitigation of the Effects of Eclipse Boundary Crossings on the Numerical Integration of Orbit Trajectories Using an Encke Type Correction Algorithm," presented as paper AAS 01-223 at the AAS/AIAA Space Flight Mechanics Meeting, Santa Barbara, CA, February 11-14, 2001.

[8] BERRY, M. and HEALY, L. "The Generalized Sundman Transformation for Propagation of High-Eccentricity Elliptical Orbits," Advances in Astronautics, San Diego, CA, February 2002.

[9] BERRY, M. M. A Variable-Step Double-Integration Multi-Step Integrator, Ph.D. thesis, Virginia Polytechnic Institute and State University, Blacksburg, Virginia, April 2004. Available at permanent link http://scholar.lib.vt.edu/theses/available/etd-04282004-071227/.

[10] HERRICK, S. Astrodynamics: Orbit Correction, Perturbation Theory, Integration, Volume 2, Van Nostrand Reinhold Company, New York, 1972.

[11] FRANKENA, J. F. "Störmer-Cowell: Straight, Summed and Split. An Overview," Journal of Computational and Applied Mathematics, 62: 129-154, 1995.

[12] WALLNER, R. N. "Earth Gravitational Error Budget Initial Report," Technical report, Kaman Sciences Corporation, 1500 Garden of the Gods Road, Colorado Springs, CO 80907, November 1994, System Memorandum KSPACE 95-18.

[13] HENRICI, P. Discrete Variable Methods in Ordinary Differential Equations, John Wiley and Sons, New York, 1962.

[14] VALLADO, D. A. Fundamentals of Astrodynamics and Applications, Volume 12 of The Space Technology Library, Microcosm Press and Kluwer Academic Publishers, El Segundo, California and Dordrecht, The Netherlands, second edition, 2001.

[15] MONTENBRUCK, O. and GILL, E. Satellite Orbits, Springer, New York, 2000.

[16] DAVIS, P. J. Interpolation and Approximation, Dover, New York, 1975.

[17] National Imaging and Mapping Agency "Department of Defense World Geodetic System 1984, its Definition and Relationships with Local Geodetic Systems," Technical report, National Imaging and Mapping Agency, 1997. Available at permanent link http://earth-info.nga.mil/GandG/ tr8350_2.html. This publication covers changes made to the standard since 1984 which are not incorporated into this software.

[18] JACCHIA, L. G. "New Static Models of the Thermosphere and Exosphere with Empirical Temperature Models," Technical Report 313, Smithsonian Astrophysical Observatory, 1970.

[19] MERSON, R. H. "Numerical Integration of the Differential Equations of Celestial Mechanics," Technical Report TR 74184, Royal Aircraft Establishment, Farnborough, Hants, UK, January 1975, Defense Technical Information Center number AD B004645. 